\section{Psicologia Escolar \\ e Educacional}

ARTIGO

DOI: http://dx.doi.org/10.1590/2175-35392021223504

Localizador - e223504

\title{
PROCRASTINAÇÃO ACADÊMICA EM ESTUDANTES UNIVERSITÁRIOS: UMA REVISÃO SISTEMÁTICA DA LITERATURA
}

\author{
Léia da Conceição Pereira ${ }^{1} \mathbb{D}$; Fabiana Pinheiro Ramos ${ }^{1} \mathbb{D}$
}

\section{RESUMO}

Procrastinação acadêmica é o adiamento de tarefas relacionadas aos estudos: realizar trabalhos, ler textos, estudar para provas, dentre outros. Este artigo teve como objetivo realizar uma revisão sistemática da literatura sobre procrastinação acadêmica em universitários, no período de 2008 a 2017. Foram escolhidas 3 bases de dados: SciELO, BvSalud e BvsPsi, com os descritores "procrastinação", "universitários", "procrastinação acadêmica" e seus correlatos em inglês e espanhol. A partir dos critérios de inclusão e exclusão foram selecionados 11 artigos completos. A maior parte dos trabalhos apontou a natureza nociva do comportamento procrastinatório, sua relação com a autorregulação da aprendizagem, bem como a diferença das estratégias de estudo empregadas por alunos procrastinadores e não procrastinadores. Concluiu-se que a pesquisa sobre a temática ainda é incipiente no contexto brasileiro necessitando de maior exploração por parte dos pesquisadores, com possíveis impactos para a compreensão do fenômeno e desenvolvimento de intervenções com estudantes do ensino superior.

Palavras-chave: autorregulação; estudantes universitários; revisão de literatura.

\section{Academic procrastination in university students: a systematic review of the literature}

\begin{abstract}
Academic procrastination is the postponement of tasks related to studies: doing work, reading texts, studying for exams, among others. This article aimed to perform a systematic review of the literature about academic procrastination in university students, from 2008 to 2017. Three databases were chosen: SciELO, BvSalud and BvsPsi, with the combination of the descriptors "procrastination", "university students", "college students", "undergraduate students", "academic procrastination" was used, as well as derivations in Portuguese and Spanish. From the inclusion and exclusion criteria, 11 complete articles were selected. Most of the works pointed out the harmful nature of procrastinatory behavior, its relationship with the self-regulation of learning, as well as the difference in the study strategies used by procrastinating and non-procrastinating students. It was concluded that research about the theme is still incipient in the Brazilian context, requiring further exploration by researchers, with possible impacts for understanding the phenomenon and developing interventions with higher education students.
\end{abstract}

Keywords: self-regulation; university students; literature review.

\section{Procrastinación académica en estudiantes universitarios: una revisión sistemática de la literatura}

\section{RESUMEN}

Procrastinación académica es la postergación de tareas relacionadas a los estudios: realizar tareas, leer textos, estudiar para exámenes, entre otros. En este artículo se tuvo como objetivo realizar una revisión sistemática de la literatura sobre procrastinación académica en universitarios, en el período de 2008 a 2017. Se eligieron 3 bases de datos: SciELO, BvSalud y BvsPsi, con los descriptores "procrastinación", "universitarios", "procrastinación académica" y sus correlatos en inglés y portugués. A partir de los criterios de inclusión y exclusión se seleccionaron 11 artículos completos. La mayor parte de los estudios apuntó la naturaleza nociva del comportamiento de procrastinación, su relación con la autorregulación del aprendizaje, así como la diferencia de las estrategias de estudio empleas por alumnos procrastinadores y no procrastinadores. Se concluyó que la investigación sobre la temática aún es incipiente en el contexto brasileño necesitando de más exploración por parte de los investigadores, con posibles impactos para la comprensión del fenómeno y desarrollo de intervenciones con estudiantes de la enseñanza universitaria.

Palabras clave: autorregulación; estudiantes universitarios; revisión de literatura.

${ }^{1}$ Universidade Federal do Espírito Santo - Vitória - ES - Brasil; cpereira.leia@gmail.com; fabiana.pinheiro.ramos@gmail.com 


\section{INTRODUÇÃO}

O ingresso no ensino superior representa uma nova configuração social e acadêmica, e implica em modificações em diversos âmbitos na vida do estudante como nos relacionamentos com familiares e amigos, no modo de percepção do mundo, no estabelecimento de novos vínculos, na organização da rotina, dentre outros (Souza, Lourenço, \& Santos, 2016). Esse momento de transição pode ser vivenciado como estressante, podendo inclusive, influenciar nos aspectos psicológicos e no rendimento acadêmico do estudante (Bardagi \& Hutz, 2011; Monteiro \& Soares, 2017). Assim, o universitário se utiliza de diversas estratégias de enfrentamento coping (Ramos, Enumo, \& Paula, 2015) com o intuito de solucionar questões emergentes nesse processo (Carlotto, Teixeira, \& Dias, 2015).

Nesse contexto, o emprego frequente de estratégias como desculpas e outros comportamentos de esquiva ao realizar uma tarefa pode ser prejudicial para a saúde e o desenvolvimento do estudante, uma vez que compromete o desempenho e o sucesso da atividade em execução (Brito \& Bakos, 2013). Como exemplos de estratégias de enfrentamento prejudiciais adotadas por universitários podem ser citados: o uso arriscado de álcool e outras drogas, procrastinação dos estudos e trabalhos, sair na noite anterior à realização de prova e exames, dentre outras. Estudos destacam a procrastinação como uma das estratégias autoprejudiciais mais utilizadas por estudantes universitários (Ganda \& Boruchovitch, 2015; Geara, Filho, \& Teixeira, 2017; Machado \& Schwartz, 2018).

A investigação científica sobre a temática da procrastinação se inicia na década de 1980 , assim, o estudo desse fenômeno é recente (Sampaio \& Bariani, 2011). A procrastinação acadêmica é definida como o adiamento desnecessário de tarefas relacionadas aos estudos, como estudar para provas, realização de trabalhos e leitura de textos (Kerbauy, 1999; Geara \& Teixeira, 2017). O estudo dessa temática é relevante socialmente, dadas as consequências que procrastinar pode acarretar na vida das pessoas, seja no cotidiano, na saúde e/ou na educação, especialmente no ensino superior, onde é requerido por parte do aluno maior senso de autonomia e autorregulação quanto ao próprio processo de aprendizagem (Ceballos, Vargas, \& Santos, 2017).

O fenômeno da procrastinação tem sido frequentemente associado à falha no processo de autorregulação (Correia \& Moura Júnior, 2017; Geara \& Teixeira, 2017; Steel \& Klingsieck, 2016). Na perspectiva de Bandura (1991), a autorregulação é compreendida enquanto processo no qual o indivíduo, de modo consciente e voluntário, gere de modo autônomo os próprios comportamentos, sentimentos e pensamentos com objetivo de alcançar suas metas. No contexto acadêmico, estudantes autorregulados se utilizam de diferentes estratégias de aprendizagem, conforme a necessidade da situação e têm capacidade de avaliar seu processo de estudo, suas limitações e suas conquistas (Martins \& Santos, 2019).

A procrastinação, como falha no processo de autorregulação, implica em descompasso entre a intenção e a ação, ou seja, há um déficit na implementação daquilo que o sujeito almeja e planeja (Sampaio, Polydoro, \& Rosário, 2012). O engajamento em comportamentos procrastinatórios no ambiente acadêmico eleva a probabilidade de experimentação de estados emocionais desagradáveis como ansiedade diante de atividades avaliativas, estresse, exaustão, redução de interesse nas atividades, dentre outros sentimentos aversivos (Lonka et al., 2014; Rabin, Fogel, \& Nutter-Upham, 2011).

Além disso, o modo como o indivíduo gerencia o tempo para efetivar determinada tarefa é relevante para a análise da procrastinação, e não somente a evitação da tarefa em si (Gouveia, Pessoa, Coutinho, Barros, \& Fonseca, 2014). Kerbauy (1999) apontou algumas relações envolvidas no comportamento de procrastinar em estudantes universitários envolvendo a variável tempo: o estudante define para si o que é a tarefa, mas acredita que o tempo para sua execução é curto; o estudante especifica a tarefa, mas considera que adiá-la é a melhor opção no momento; o estudante supõe que novo prazo será concedido ou que terá nova oportunidade de completar a tarefa; o estudante inicia a tarefa, mas a interrompe e prorroga seu término; ou ainda, o estudante prorroga o início da tarefa e superestima o tempo disponível para fazê-la.

A literatura da área discute, ainda, os efeitos negativos da procrastinação no processo de aprendizagem, desempenho acadêmico, senso de controle e bemestar subjetivo dos estudantes (Geara \& Teixeira, 2017; Grunschel, Partrzek, \& Fries, 2013). O emprego prolongado da procrastinação pode contribuir para o desenvolvimento de quadros clínicos como depressão, ansiedade extrema, estresse, comprometimento da atenção e instabilidade emocional (Morris \& Fritz, 2015; Patrzek, Grunschel, \& Fries, 2012; Steel \& Klingsieck, 2016). Com relação aos efeitos nocivos da procrastinação, universitários participantes dos estudos desenvolvidos por Klassen, Krawchuk e Rajani (2008) e Klassen e Kuzucu (2009) atribuíram ao elevado índice de procrastinação: a má qualidade dos trabalhos acadêmicos, a redução da possibilidade de atingir objetivos acadêmicos com êxito, e a tendência a desempenho acadêmico inferior ao esperado. Nesse contexto, destaca-se a importância de serviços de apoio psicológico ao estudante que possam auxiliálos na organização dos estudos, na autorregulação da aprendizagem e na redução da procrastinação (Ramos 
et al., 2018).

Tendo em vista a importância da procrastinação no contexto acadêmico, bem como seus efeitos prejudiciais aos estudantes, este trabalho teve como objetivo realizar uma revisão da literatura acerca da produção científica de artigos com foco em aspectos envolvidos no fenômeno da procrastinação acadêmica em universitários.

\section{MÉTODO}

Trata-se de uma revisão sistemática de literatura, cujo objetivo foi o levantamento de artigos que apontassem possíveis indicadores ou fatores contribuintes para o fenômeno da procrastinação em contexto acadêmico. Após breve busca assistemática, verificou-se a escassez de publicações sobre a temática em língua portuguesa e grande número de publicações em espanhol. Desse modo, a fim de ampliar o escopo da busca, utilizouse a combinação dos descritores "procrastinação", "universitários", "procrastinação acadêmica", bem como derivações na língua inglesa e espanhola: "procrastination", "university students", "college students", "undergraduate students", "academic procrastination", "procrastinación", "universitarios" e "procrastinación académica".

Adotou-se como critérios de inclusão artigos científicos completos e gratuitos, publicados no período de 2008 a 2017, dispostos nas bases de dados: SciELO, Biblioteca Virtual em Saúde (BvSalud), Biblioteca Virtual em Saúde - Psicologia (Bvs-Psi) que tratassem do fenômeno da procrastinação acadêmica em universitários. Excluíram-se teses, dissertações, capítulos de livros, artigos repetidos, bem como artigos pagos, incompletos, em outros idiomas e os que não abordavam o assunto de interesse. Foram encontradas 218 publicações no levantamento inicial, realizado entre abril e maio de 2018. Após leitura dos resumos, verificou-se que apenas 11 trabalhos atendiam aos critérios de inclusão.

Os artigos selecionados foram analisados e categorizados da seguinte forma: (a) Características gerais dos artigos: autores, ano de publicação, base de dados, local da publicação, idioma, país de filiação institucional do autor; (b) Características metodológicas: artigo teórico ou empírico, e tipo de delineamento; e (c) Tópico temático: variáveis avaliadas em função da procrastinação acadêmica e possíveis preditores da procrastinação acadêmica.

\section{RESULTADOS E DISCUSSÃO}

Os 11 artigos encontrados, bem como seus autores, ano de publicação e principais preditores da procrastinação acadêmica em universitários podem ser vistos na Tabela 1.

Dentre os artigos selecionados e analisados, obser- vou-se o maior número de publicações junto à base de dados SciELO ( $N=8)$, seguido de BvSalud ( $N=3$ ) e, por fim, nenhuma publicação foi localizada na base de dados Bvs-Psi. Identificaram-se 10 artigos empíricos $(90,9 \%)$ e apenas um artigo teórico (9,09\%). O maior número de publicações de cunho empírico pode ser explicado pela potencialidade desse tipo de artigo em fornecer dados, suscitar reflexões e contribuir ativamente para investigações posteriores. Apesar da relevância de artigos teóricos, notou-se a escassez desse tipo de trabalho. A única produção desse cunho encontrada acerca da temática da procrastinação acadêmica foi uma revisão integrativa de literatura (Brito \& Bakos, 2013), o que indica uma lacuna e sinaliza a necessidade de maior atenção por parte da comunidade científica, pois a revisão sistemática possibilita a síntese da produção de conhecimento sobre determinado tema.

Apesar do quantitativo reduzido de artigos encontrados acerca dos preditores da procrastinação acadêmica, o número de publicações mostrou-se crescente no intervalo temporal delimitado nesse trabalho, conforme pode ser visto na Tabela 2. Esse dado pode estar relacionado ao fato de a investigação da temática ser relativamente recente na literatura em geral, conforme apontado por Sampaio e Bariani (2011), e sinaliza a necessidade de maior compreensão do fenômeno (Machado \& Schwartz, 2018), sobretudo para a consolidação dessa temática, ainda incipiente no Brasil.

Em relação ao local de publicação dos artigos, obteve-se: Colômbia $(N=4)$, Brasil $(N=2)$, Estados Unidos ( $N=2)$, Chile $(N=1)$, Espanha $(N=1)$ e Peru ( $N=$ 1). Alguns trabalhos, embora produzidos originalmente em países como México, Equador, Argentina, Irã e Canadá, foram publicados em revistas científicas de outros países, dentre eles Colômbia, Peru, Chile e Estados Unidos. Esses resultados indicam uma produção majoritária realizada em países latinoamericanos, o que corresponde a $72,7 \%$ do total dos artigos publicados ( $\mathrm{N}$ $=8)$, contra $18,1 \%(\mathrm{~N}=2)$ de trabalhos publicados em revistas dos Estados Unidos e 9,09\% ( $N=1)$ em revista da Espanha. Quanto ao idioma, a maior parte dos artigos foi redigido em espanhol $(N=6)$, seguido de inglês $(N=$ 4) e apenas 1 em língua portuguesa.

Observou-se um número de publicações pouco expressivo em contexto brasileiro no âmbito das bases de dados especificadas para a realização desse estudo, sendo um artigo publicado em língua portuguesa e um em língua inglesa. Esse achado corrobora com levantamento realizado em 2016 por Geara e Teixeira (2017), que também indicou a procrastinação acadêmica como tema pouco explorado. Os referidos autores utilizaram o descritor "procrastinação" em oito bases de dados previamente selecionadas e localizaram 15 estudos diferentes com publicação em língua portuguesa, cujo 
Tabela 1. Artigos Sobre Procrastinação Acadêmica Em Universitários Encontrados Na Revisão (N=11).

\begin{tabular}{|c|c|c|}
\hline Autores & Ano & Possíveis preditores da procrastinação acadêmica \\
\hline Brito e Bakos & 2013 & $\begin{array}{l}\text { Crenças disfuncionais, insatisfação, baixa realização } \\
\text { acadêmica, baixa autorregulação }\end{array}$ \\
\hline Ceballos, Vargas e Santos & 2017 & $\begin{array}{l}\text { Procrastinação como estilo interativo do sujeito independente } \\
\text { do alto ou baixo nível de exigência da tarefa }\end{array}$ \\
\hline Dominguez-Lara e Campos-Uscanga & 2017 & Satisfação com os estudos não foi preditora da procrastinação \\
\hline Furlan & 2013 & Baixa autoeficácia regulatória e ansiedade frente a exames \\
\hline Ganda e Boruchovitch & 2015 & Idade (mais jovens) e gênero (mulheres) \\
\hline $\begin{array}{l}\text { González-Brignardello e Sánchez-Elvira- } \\
\text { Paniagua }\end{array}$ & 2013 & $\begin{array}{l}\text { Baixo engajamento, estratégia de estudo deficiente, baixa } \\
\text { autorregulação }\end{array}$ \\
\hline Muñoz-Olano e Hurtado-Parrado & 2017 & $\begin{array}{l}\text { Não esclarecimento de objetivos acadêmicos (definição de } \\
\text { metas e prazos) }\end{array}$ \\
\hline Rahimi, Hall e Pychyl & 2016 & Relação entre atrasos, culpabilidade e responsabilidade moral \\
\hline $\begin{array}{l}\text { Ramos-Galarza, Jadán-Guerrero, Paredes- } \\
\text { Nuñez, Bolaños-Pasquel e Gómez-García }\end{array}$ & 2017 & Vício em internet e baixo rendimento acadêmico \\
\hline Rodríguez e Clariana & 2017 & Idade (mais jovens) \\
\hline Vahedi, Farrokhi, Gahramani e Issazadegan & 2012 & Baixa autorregulação metacognitiva \\
\hline
\end{tabular}

Fonte: elaboração própria.

Tabela 2. Quantitativo De Artigos Sobre Procrastinação Acadêmica, Por Ano, Encontrados Na Revisão ( $N=11$ ).

\begin{tabular}{lc}
\hline Ano de publicação & Número de artigos \\
\hline 2017 & 5 \\
2016 & 1 \\
2015 & 1 \\
2014 & 0 \\
2013 & 3 \\
2012 & 1 \\
1998 a 2011 & 0 \\
\hline
\end{tabular}

Fonte: Elaboração própria.

conteúdo associava a procrastinação com temáticas da área da Psicologia, Educação, Administração e Economia. Ao buscar por "procrastination" na base Psyclnfo esses autores geraram 937 resultados incluindo inúmeros instrumentos sobre a temática em inglês, o que indica maior prevalência dessa temática na literatura internacional.

Cabe mencionar que outra lacuna observada durante o processo de levantamento dos artigos que embasaram esta revisão foi que o único instrumento encontrado foi a "Escala de Procrastinação Ativa" validada por Gouveia, Pessoa, Coutinho, Barros e Fonseca (2014). Em síntese, nota-se que existem poucas pesquisas nacionais que investiguem de modo sistematizado a procrastinação acadêmica e desse modo, pode-se hipotetizar que: a) poucos pesquisadores brasileiros têm se dedicado a estudar de modo sistematizado a temática; b) trabalhos sobre este tema são desenvolvidos, mas estão sendo pouco publicados; ou ainda que, c) há um número significativo de trabalhos abordando a temática no Brasil, se o escopo das bases de dados utilizadas for ampliado. Novas pesquisas de revisão poderão elucidar quais dentre essas hipóteses são verdadeiras. Não obstante, a temática da procrastinação parece ainda não ter despertado interesse por parte dos estudiosos e ainda carecer de exploração sistemática na literatura nacional, conforme apontado anteriormente por Brito e Bakos (2013) e Ceballos, Vargas e Santos (2017).

Além do aspecto relacionado ao quantitativo de publicações, é possível avaliar algumas discussões teóricas presentes nos artigos levantados sobre a análise da procrastinação. Os artigos analisados possibilitaram a identificação da idade como um fator importante: algumas pesquisas evidenciaram que adolescentes e alunos mais jovens têm maior tendência a engajar-se em comportamentos procrastinatórios em função da dificuldade de rejeitar tentações (menor autocontrole) e menor senso de orientação futura, uma vez que ficam mais sob controle de recompensas de curto prazo do que de longo prazo (Ganda \& Boruchovitch, 2015; Rodriguez \& Clariana, 2017). Estudantes mais velhos, por sua vez, tenderiam a ter maior autocontrole e maior senso de perspectiva futura consolidado; assim, a procrastinação tenderia a diminuir com a idade, pois, após inúmeras vivências de experiências negativas e 
dolorosas, os indivíduos adquiririam, com o tempo, hábitos de estudos mais adaptativos de modo a se esquivar de experiências aversivas decorrentes da procrastinação (Ganda \& Boruchovitch, 2015; Rodríguez \& Clariana, 2017).

Um dos aspectos mais relacionados à procrastinação acadêmica na literatura obtida foram as dificuldades relacionadas à autorregulação, presente em 27,2\% dos artigos levantados (Brito \& Bakos, 2013; GonzálezBrignardello \& Sánchez-Elvira-Paniagua, 2013; Vahedi, Farrokhi, Gahramani, \& Issazadegan, 2012). Estudiosos têm concebido a autorregulação enquanto processo consciente, autodirigido, que engloba autonomia e estratégias orientadas por critérios e metas de aprendizagem (Bandura, 1991). Esse constructo está intimamente relacionado ao controle e regulação do aluno acerca do próprio comportamento, pensamentos, motivação, cognição em contexto acadêmico (Machado \& Schwartz, 2018; Rabin et al., 2011). Pesquisas têm considerado a procrastinação enquanto falha relacionada ao processo de autorregulação da aprendizagem (Steel \& Klingsieck, 2016; Geara \& Teixeira, 2017); em síntese: quanto mais eficiente for a autorregulação, menor a tendência em procrastinar (Sampaio et al., 2012).

Os estudos analisados para fins dessa revisão também convergiram quanto ao aspecto nocivo do comportamento procrastinatório e quanto ao fato dos universitários procrastinadores utilizarem estratégias de estudos deficitárias, que implicam em: dificuldades em estabelecer objetivos, dificuldades de se planejar, percepção deficitária quanto à própria capacidade de realização de atividades acadêmicas, menor interesse em relação às atividades, e vivência de emoções negativas e intensas (Dominguez-Lara \& CamposUscanga, 2017; Muñhoz-Olano \& Hurtado-Parrado, 2017; Rahimi, Hall, \& Pychyl, 2016). Observou-se na literatura analisada a relação da procrastinação com aspectos emocionais (e.g. ansiedade e insatisfação), aspectos cognitivos (e.g. crenças disfuncionais e baixa autoeficácia), e aspectos comportamentais como dificuldade em manejar o tempo, baixo engajamento e baixo rendimento acadêmico (Brito \& Bakos, 2013; Furlan, 2013; González-Brignardello \& Sánchez-ElviraPaniagua, 2013; Ramos-Galarza, Jadán-Guerrero, Paredes-Núñez, Bolaños-Pasquel, \& Gómez-García, 2017).

A procrastinação acadêmica, se vivenciada de forma prolongada ou intensa, pode contribuir para o desenvolvimento de problemas clínicos como estresse, ansiedade e estilo de vida não saudável (Lonka et al., 2014). Desse modo, conhecer os múltiplos componentes implicados no fenômeno contribui tanto para a produção e ampliação de conhecimento na área, como possibilita o desenvolvimento de tecnologias comportamentais de intervenção voltadas à minimização do problema. Além disso, o desenvolvimento de estratégias de intervenção focadas no processo de autorregulação da aprendizagem é relevante, uma vez que o estudante dotado de boa capacidade de autorregulação emprega estratégias de estudos mais adequadas e eficientes, avalia o próprio processo de aprendizagem de forma mais fidedigna e possui maior senso de autoeficácia (Wolters, 2003), diminuindo a procrastinação.

\section{CONCLUSÃO}

O objetivo desta revisão sistemática foi o levantamento de artigos que abordassem possíveis indicadores ou fatores contribuintes para a procrastinação em universitários. Constatou-se que a temática carece de estudos sistematizados no contexto brasileiro que se proponham a analisar a especificidade desse fenômeno no âmbito acadêmico. Além disso, parece haver escassez de instrumentos validados no país para avaliar tal constructo. Tais lacunas merecem atenção por parte de pesquisadores brasileiros, dadas as consequências nocivas desse fenômeno em grau crônico ao desempenho e à saúde mental dos indivíduos. Sugere-se a realização de novos trabalhos do tipo revisão sistemática que incorporem maior quantitativo de bases de dados, a fim de avaliar melhor a prevalência do tema nos estudos feitos por pesquisadores brasileiros.

Neste sentido, a compreensão dos mecanismos implicados na procrastinação acadêmica configura-se como temática relevante para realização de futuras pesquisas, uma vez que possibilita o desenvolvimento de intervenções de cunho preventivo com foco no bemestar psicológico de estudantes do ensino superior. Tais pesquisas poderão contribuir com a elaboração de políticas de assistência estudantil focadas na promoção da qualidade de vida e prevenção das situações crônicas vivenciadas por estudantes de ensino superior, como parece ser o caso da procrastinação.

\section{REFERÊNCIAS}

Bandura, A. (1991). Selfregulation of motivation through anticipatory and selfreactive mechanisms. In Dienstbier, R. A. (Ed.), Perspectives on motivation: Nebraska symposium on motivation (pp. 69164). Lincoln: University of Nebraska Press.

Bardagi, M. P.; Hutz, C. S. (2011). Eventos estressores no contexto acadêmico: Uma breve revisão da literatura brasileira. Interação em Psicologia, 15(1), 111-119.

Brito, F. S.; Bakos, D. G. S. (2013). Procrastinação e terapia cognitivo-comportamental: Uma revisão integrativa. Revista Brasileira de Terapias Cognitivas, 9(1), 34-41.

Carlotto, R. C.; Teixeira, M. A. P.; Dias, A. C. G. (2015). Adaptação acadêmica e coping em estudantes universitários. PsicoUSF, 20(3), 421-432. Recuperado de DOI: http://dx.doi. 


\section{org/10.1590/1413-82712015200305}

Ceballos, C. G. T.; Vargas, M. A. P.; Santos, C. V. (2017). El estudio de la procrastinación humana como un estilo interactivo. Avances en Psicología Latinoamericana, 35(1), 153-163. Recuperado de DOI: http://dx.doi.org/10.12804/ revistas.urosario.edu.co/apl/a.4330

Correia, R. R.; Moura Júnior, P. J. (2017). Aprendizagem e procrastinação: Uma revisão de publicações no período de 2005 a 2015. Revista Iberoamericana sobre Calidad, Eficacia y Cambio en Educación, 15(2), 111-128. Recuperado de DOI: doi:10.15366/reice2017.15.2.006

Dominguez-Lara, S. A.; Campos-Uscanga, Y. (2017). Influencia de la satisfacción con los estudios sobre la procrastinación académica en estudiantes de psicología: Un estudio preliminar. Liberabit, 23(1), 123-135. Recuperado de DOI: http://dx.doi.org/https://doi.org/10.24265/liberabit.2017. v23n1.09

Furlan, L. A. (2013). Eficacia de una intervención para disminuir la ansiedad frente a los exámenes en estudiantes universitarios argentinos. Revista Colombiana de Psicología, 22(1), 75-89.

Ganda, D. R.; Boruchovitch, E. (2015). Self-handicapping strategies for learning of preservice teachers. Estudos de Psicologia (Campinas), 32(3), 417-425. Recuperado de DOI: https://doi.org/10.1590/0103-166X2015000300007

Geara, G. B.; Filho, N. H.; Teixeira, M. A. P. (2017). Construção da escala de motivos de procrastinação acadêmica. Psico (Porto Alegre), 48(2), 140-151. Recuperado de DOI: https:// doi.org/10.15448/1980-8623.2017.2.24635

Geara, G. B.; Teixeira, M. A. P. (2017). Questionário de procrastinação acadêmica - consequências negativas: Propriedades psicométricas e evidências de validade. Avaliação Psicológica, 16(1), 59-69. Recuperado de DOI: 10.15689/ap.2017.1601.07

González-Brignardello, M. P.; Sánchez-Elvira-Paniagua, A. (2013). ¿Puede amortiguar el engagement los efectos nocivos de la procrastinación académica? Acción Psicológica, 10(1), 117-134.

Gouveia, V. V.; Pessoa, V. S.; Coutinho, M. L.; Barros, I. C. S.; Fonseca, A. A. (2014). Escala de Procrastinação Ativa: Evidências de validade fatorial e consistência interna. Psico-USF, 19(2), 345-354.

Grunschel, C.; Patrzek, J.; Fries, S. (2013). Exploring reasons and consequences of academic procrastination: An interview study. European Journal of Psychology of Education, 28(3), 841-861.

Kerbauy, R. (1999). Procrastinação: Adiamento de tarefas. In: R. A. Banaco (Ed.), Sobre o comportamento e cognição: Aspectos teóricos, metodológicos e de formação em análise do comportamento e terapia cognitivista (Vol. 1, pp. 445451). Santo André, SP: ESETec Editores Associados.

Klassen, R. M.; Krawchuk, L. L.; Rajani, S. (2008). Academic procrastination of undergraduates: Low self-efficacy to self-regulate predicts higher levels of procrastination. Contemporary Educational Psychology, 33(4), 915-931.
Klassen, M. R.; Kuzucu, E. (2009). Academic procrastination and motivation of adolescents in Turkey. Educational Psychology, 29(1), 69-81.

Lonka, K.; Chow, A.; Keskinen, J.; Hakkarainen, K.; Sandström, N.; Pyhältö, K. (2014). How to measure PhD. students' conceptions of academic writing, and are they related to wellbeing? Journal of Writing Research, 5(3), 245-269.

Machado, B. A. B.; Schwartz, S. (2018). Procrastinação e aprendizagem acadêmica. Revista Eletrônica Científica da UERGS, 4(1), 119-135. Recuperado de DOI: https://doi. org/10.21674/2448-0479.41.119-135

Martins, R. M. M.; Santos, A. A. A. (2019). Estratégias de aprendizagem e autoeficácia acadêmica em universitários ingressantes: Estudo correlacional. Psicologia Escolar e Educacional, 23, e176346. Recuperado de DOI: https:// doi.org/10.1590/2175-35392019016346.

Monteiro, M. C.; Soares, A. B. (2017). Concepções de estudantes sobre resolução de problemas e automonitoria. Psicologia Escolar e Educacional, 21(2), 333-341. Recuperado de DOI: https://doi.org/10.1590/2175-3539201702121124.

Morris, P. E.; Fritz, C. O. (2015). Conscientiousness and procrastination predict academic coursework marks rather than examination performance. Learning and Individual Differences, 39,193-198. Recuperado de DOI: https://doi. org/10.1016/j.lindif.2015.03.007

Muñoz-Olano, J. F.; Hurtado-Parrado, C. (2017). Effects of goal clarification on impulsivity and academic procrastination of college students. Revista Latinoamericana de Psicología, 49(3), 173-181. Recuperado de DOI: https://doi. org/10.1016/j.rlp.2017.03.001

Patrzek, J.; Grunschel, C.; Fries, S. (2012). Academic procrastination: The perspective of university counsellors. International Journal for the Advancement of Counselling, 34(3), 185-201.

Rabin, L. A.; Fogel, J.; Nutter-Upham, K. E. (2011). Academic procrastination in college students: The role of selfreported executive function. Journal of Clinical and Experimental Neuropsychology, 33(3), 344-357.

Rahimi, S.; Hall, N. C.; Pychyl, T. A. (2016). Attributions of responsibility and blame for procrastination behavior. Frontiers in Psychology, 7:1179. Recuperado de DOI: https://doi.org/10.3389/fpsyg.2016.01179

Ramos, F. P.; De Andrade, A. L.; Jardim, A. P.; Ramalhete, J. N. L.; Pirola, G. P.; Egert, C. (2018). Intervenções psicológicas com universitários em serviços de apoio ao estudante. Revista Brasileira de Orientação Profissional, 19(2), 221232. Recuperado de DOI: http://dx.doi.org/1026707/19847270/2019v19n2p221

Ramos, F. P.; Enumo, S. R. F.; Paula, K. M. P. (2015). Teoria Motivacional do Coping: Uma proposta desenvolvimentista de análise do enfrentamento do estresse. Estudos de Psicologia (Campinas), 32(2), 269-279. Recuperado de DOI: https://doi.org/10.1590/0103-166X2015000200011

Ramos-Galarza, C.; Jadán-Guerrero, J.; Paredes-Núñez, L.; Bolaños-Pasquel, M.; Gómez-García, A. (2017). 
Procrastinación, adicción al internet y rendimiento académico de estudiantes universitarios ecuatorianos. Estudios Pedagógicos (Valdivia), 43(3), 275-289. Recuperado de DOI: https://doi.org/10.4067/S071807052017000300016

Rodríguez, A.; Clariana, M. (2017). Procrastinación en estudiantes universitarios: Su relación con la edad y el curso académico. Revista Colombiana de Psicología, 26(1), 45-60. Recuperado de DOI: 10.15446/rcp.v26n1.53572

Sampaio, R. K. N.; Bariani, I. C. D. (2011). Procrastinação acadêmica: Um estudo exploratório. Estudos Interdisciplinares em Psicologia, 2(2), 242-262.

Sampaio, R. K. N.; Polydoro, S.; Rosário, P. (2012). Autorregulação da aprendizagem e a procrastinação acadêmica em estudantes universitários. Cadernos de Educação, 42, 119-142.
Souza, L. K; Lourenço, E.; Santos, M. R. G. (2016). Adaptação à universidade em estudantes ingressantes na graduação em psicologia. Psicologia da Educação, 42, 35-48. Recuperado de DOI: http://dx.doi.org/10.5935/2175-3520.20150023

Steel, P.; Klingsieck, K. B. (2016). Academic procrastination: Psychological antecedents revisited. Australian Psychologist, 51(1), 3646. Recuperado de DOI: https:// doi.org/10.1111/ap.12173

Vahedi, S.; Farrokhi, F.; Gahramani, F.; Issazadegan, A. (2012). The relationship between procrastination, learning strategies and statistics anxiety among Iranian college students: A canonical correlation analysis. Iranian Journal of Psychiatry and Behavioral Sciences, 6(1), 40-46.

Wolters, C. A. (2003). Understanding procrastination from a self-regulated learning perspective. Journal of Education Psychology, 95(1), 179-187.

Recebido: 03 de maio de 2019

Aprovado: 16 de abril de 2020 